\title{
DETERMINAÇÃO SIMULTÂNEA DE ANTOCIANINAS E COMPOSTOS FENÓLICOS TOTAIS EM FRUTOS INTACTOS DE JAMBO (EUGENIA MALACCENSIS) EMPREGANDO A ESPECTROSCOPIA NO INFRAVERMELHO PRÓXIMO (NIR) E REGRESSÃO POR MÍNIMOS QUADRADOS PARCIAIS (PLS2)
}

\author{
T. R. VIEGAS ${ }^{1}$, M. M. L. DUARTE${ }^{1}$, M. F. N. MONTEIRO ${ }^{1}$, W. R. Q. PEGADO ${ }^{1}$, A. L. M. L. \\ MATA $^{1}$, ' K. M. G de LIMA ${ }^{2}$ \\ ${ }^{1}$ Universidade Federal do Rio Grande do Norte (UFRN), Departamento de Engenharia Química. \\ ${ }^{2}$ Universidade Federal do Rio Grande do Norte (UFRN), Grupo de Pesquisa em Química \\ Biológica e Quimiometria, Instituto de Química. \\ E-mail para contato: thaynaviegas@gmail.com
}

RESUMO - O objetivo deste trabalho foi utilizar a potencialidade da espectroscopia no infravermelho próximo (NIRS) como um método rápido e não destrutivo para determinação simultânea de antocianinas (AT) e compostos fenólicos totais (CFT) em jambos intactos. Amostras de jambo com teor de AT variando 9,07 a 218,7 mg L $\mathrm{m}^{-1}$ e CFT totais entre 31,6 a 229,02 foram adquiridas na região metropolitana de Natal-Brasil, e foram coletados espectros NIR no intervalo de 1000-2500 nm utilizando um espectrofotômetro Antaris MX FT-NIR (Thermo Fisher Scientific Inc., USA) equipado com uma sonda de transflectância. A influência de alguns pré-processamentos espectrais (suavização Savitzky-Golay, correção multiplicativa de espalhamento de luz (MSC) e derivadas Savitzky-Golay) e calibração multivariada usando o algoritmo de regressão por mínimo quadrados parciais (PLS-2) foram calculados em ambiente Matlab 7.0 (MathWorks, Natick, Massachusetts, USA), especificamente usando o pacote do PLS-toolbox (Eigenvector Research, Inc., Wenatchee, WA, USA, version 6.01). Modelos de validação para o teor de AT e CFT tiveram um coeficiente de correlação $\left(R^{2}\right)$ de 0,85 e 0,92 , com erro médio quadrado de previsão (RMSEP) de 22,34 e 15,07, respectivamente. A partir desses resultados, conclui-se que a espectroscopia NIR pode ser usada como uma técnica não destrutiva para determinação simultânea de AT e CFT em jambos.

\section{INTRODUÇÃO}

O jambo vermelho é um fruto cultivado em praticamente todo o Brasil em áreas de climas quentes e úmidos. O jambo é pouco explorado, sendo seu consumo praticamente todo in natura. Isto provoca um grande desperdício em sua época de safra, instigando assim pesquisas sobre o potencial tecnológico deste fruto (Silva et al, 2012). A pigmentação natural presente na casca do jambo indica a presença de antocianinas, pertencentes ao grupo dos flavonóides, que são encontrados apenas em vegetais e apresentam cores que variam de vermelho intenso ao violeta e 
azul (Ribeiro \& Seravalli, 2007). A utilização de corantes na indústria de alimentos é uma prática comum, já que a cor e a aparência tem um papel importante na aceitação dos produtos pelo consumidor (Lopes et al, 2007). Riscos toxicológicos em relação aos corantes sintéticos amplamente utilizados na indústria de alimentos são alvos de preocupações da atualidade, logo, pesquisas vêm sendo desenvolvidas visando a substituição por corantes naturais, como o extraído do jambo, ou até mesmo a eliminação de corantes sintéticos no processamento de alimentos.

Nos últimos anos, foram desenvolvidos métodos para determinar as antocianinas em frutos, tais como UV-Vis, ressonância magnética nuclear (Missang et al., 2003), espectrometria de massa (Williams et al., 2002) e electroforese de capilaridade (Bednár et al., 2005). Apesar da confiabilidade, estes métodos destroem o fruto, produzem resíduos químicos e exigem tempo e/ou investimento elevado para a realização das análises.

Tendo em vista as desvantagens dos métodos existentes, a aceitação do NIR tem aumentado em vários campos analíticos durante a última década (Neves et al., 2012). A vantagem desta análise se dá pelo fato de não ser necessária uma preparação prévia do fruto, sendo possível a determinação de antocianinas a partir de frutos intactos (Zude et al., 2011). Além disso, o NIR pode analisar vários parâmetros utilizando apenas uma medida. A literatura não registra calibração do teor de antocianina total e compostos fenólicos totais pela espectroscopia NIR no jambo, ou nenhuma outra técnica rápida e não destrutiva.

As limitações do NIR dizem respeito à alta umidade, o tamanho e a forma irregular dos frutos. Certos frutos possuem a casca grossa de difícil penetração. Para contornar estas dificuldades, algoritmos quimiométricos são aplicados aos dados, como a regressão pelo método de mínimos quadrados parciais (PLS2) entre outros. Basicamente, estes algoritmos eliminam variáveis que não se relacionam diretamente com a propriedade de interesse, como aquelas que adicionam apenas ruídos, não-linearidades, ou informações irrelevantes. Eles também eliminam possíveis interferências e variáveis que geram uma relação baixa de sinal/ruído, o que é indicativo de uma baixa sensibilidade. (Costa \& de Lima, 2013)

O objetivo deste estudo foi avaliar a potencialidade da espectroscopia do infravermelho próximo (NIR) e PLS2 como um método rápido e não destrutivo para a determinação simultânea de antocianinas e compostos fenólicos totais em jambo intactos coletados de acordo com a disponibilidade de safra em Natal (Rio Grande do Norte, Brasil).

\section{METODOLOGIA}

\subsection{Amostras}

Pelo método de referência, os frutos foram divididos em grupos de dez jambos com genótipos semelhantes. Em seguida armazenados a temperatura ambiente antes de realizar as medidas no NIR. As análises pelo método de referência foram realizadas no mesmo dia ou no dia seguinte. As propriedades morfológicas das amostras, incluindo as massas médias e diâmetros 
médios, foram medidas antes das análises.

\subsection{Método de referência e Instrumentação}

Os extratos foram obtidos pelo método de extração sequencial de Azevedo (2010). O extrato de cada grupo de frutos foi obtido em reator enjaquetado, com agitação e temperatura controladas utilizando valores dos parâmetros ótimos obtidos no estudo de Brito (2009).

Para a análise das antocianinas totais foi empregado o método do $\mathrm{pH}$ diferencial (Giusti \& Wrolstad, 2001). O extrato foi diluído em soluções tampão de cloreto de potássio $(\mathrm{pH}=1,0)$, e carbonato de sódio $(\mathrm{pH}=4,5)$, com o fator de diluição $=10$ ( $2 \mathrm{~mL}$ de amostra/0,2 mL extrato). Logo, 0,2 $\mathrm{mL}$ de extrato foram adicionados a $1,8 \mathrm{~mL}$ de cada tampão, posteriormente homogeneizados em agitador vórtex e deixados em equilíbrio à temperatura ambiente em câmara escura por 30 minutos. O branco foi preparado da mesma maneira, substituindo-se o extrato por água destilada. A absorbância foi medida nos comprimentos de onda de $510 \mathrm{~nm}\left(\mathrm{~A}_{1}\right)$ e a $700 \mathrm{~nm}$ $\left(\mathrm{A}_{2}\right)$ para corrigir o espalhamento de luz produzido por uma possível turbidez da amostra. A absorbância resultante e o cálculo da concentração das antocianinas são calculados a partir das equações (1) e (2):

$$
\begin{aligned}
& A=(A 1-A 2)_{p H 1,0}-(A 1-A 2)_{p H 4,5} \\
& C=\frac{(A \cdot F D \cdot M M \cdot 1000)}{(s \cdot b)}
\end{aligned}
$$

onde C é a concentração de antocianinas (mg/L); A é a absorbância; FD é o fator de diluição; MM é a massa molar do padrão $(\mathrm{g} / \mathrm{mol})$; $\varepsilon$ é o coeficiente de absortividade molar do padrão ( $\mathrm{L} / \mathrm{cm} \mathrm{mol})$ e b é o caminho óptico $(\mathrm{cm})$.

O teor de fenólicos totais nos extratos obtidos foi determinado segundo a metodologia descrita por Cheplick et al. (2010). Em um tubo de ensaio, foram colocados $1 \mathrm{~mL}$ do extrato, 1 $\mathrm{mL}$ de etanol 95\%, $5 \mathrm{~mL}$ de água destilada e $0,5 \mathrm{~mL}$ do reagente Folin-Ciocalteau (RFC). A solução preparada foi homogeneizada e deixada em repouso durante 5 minutos. Em seguida, adicionou-se $1 \mathrm{~mL}$ de carbonato de sódio 5\% e esta foi mantida em câmara escura por 60 minutos. Posteriormente as amostras foram novamente homogeneizadas e suas absorbâncias foram medidas a $725 \mathrm{~nm}$. O branco foi preparado seguindo o mesmo procedimento, porém substituindo o extrato por solução etanólica 95\%. Para os resultados de CFT em microgramas equivalentes de ácido gálico por $\mathrm{mL}$ de extrato ( $\mathrm{ppm}$ ) foi utilizada uma curva de calibração, com a finalidade de converter as absorbâncias obtidas das amostras (Azevedo, 2010). Todas as medidas foram feitas em triplicatas para cada grupo. Através da curva de calibração obtém-se a equação (3). 


\section{9 a 22 de outubro de 2014 \\ Florianópolis/SC}

As medidas dos espectros foram realizadas utilizando o espectrofotômetro Antaris MX FTNIR (Thermo Fisher Scientific Inc, Madison, WI, USA) equipado com uma sonda de transflectância. Os espectros NIR foram coletados no intervalo de $1000-2500 \mathrm{~nm}$, com leituras em quatro posições distintas, como mostrado na Figura 1. A resolução espectral utilizada foi de $8 \mathrm{~cm}^{-1}$ e 100 varreduras por espectro. A partir do valor médio das quatro leituras foi obtido um espectro médio para cada amostra. O espectro de politetrafluoretileno (PTFE) foi utilizado como background.
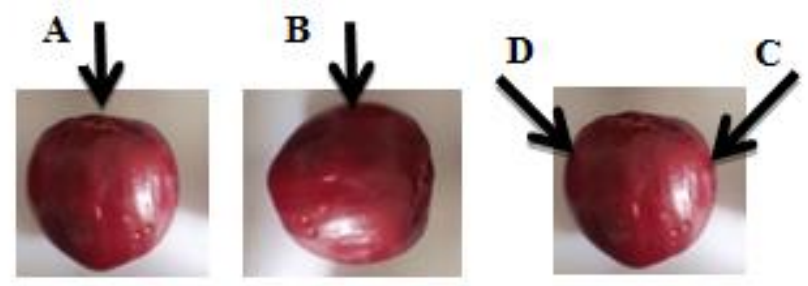

Figura 1 - Posições de aquisição espectral NIR dos frutos intactos de Jambo.

\subsection{Análise dos dados}

A análise dos dados foi realizada utilizando o MATLAB versão 7.0 (Math-Works, Natick, Massachusetts, USA), especificamente o pacote do PLS-toolbox (Eigevector Research, Inc., Wenatchee, WA, USA, version 6.01). Diversos métodos de pré-processamento foram aplicados para a matriz de dados obtidos, incluindo a suavização e derivada Savitzky-Golay bem como o método de correção multiplicativa de espalhamento de luz (MSC). O alisamento de Savitzky-Golay trata-se de um método matemático de suavização, baseado em uma regressão polinomial, tendo como objetivo a eliminação de ruídos. MSC é um método utilizado para compensar efeitos aditivos e/ou multiplicativos dos espectros de reflectância. O desempenho destas técnicas de pré-processamento foi comparado baseado na calibração PLS2 para as antocianinas totais e compostos fenólicos totais, simultaneamente. As amostras foram divididas em dois subconjuntos de dados, um para a calibração e outro para a previsão externa através do algoritmo Kennard Stone (KS) aplicado aos espectros NIR. Para os modelos de calibração multivariadas, o menor erro é obtido quando se utiliza o número ótimo de fatores do PLS, que é encontrado utilizando a variância da matriz das respostas instrumentais. Os resultados previstos foram utilizados pra testar a capacidade preditiva dos modelos PLS2 para os dois parâmetros simultaneamente.

\section{RESULTADOS E DISCUSSÃO}

O espectro médio de reflectância difusa com leituras nas posições diferentes do jambo obtido na região de 1000 a $2500 \mathrm{~nm}$ está apresentado na Figura 2. Como pode ser visualizado nesta figura, os espectros apresentam ruídos instrumentais, desvio de linha de base, efeitos de 


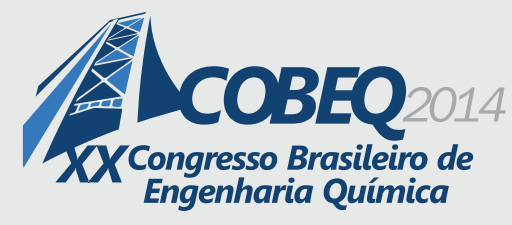

19 a 22 de outubro de 2014
Florianópolis/SC

espalhamento de luz, que provocam o deslocamento do espectro na vertical, e finalmente, espalhamentos multiplicativos. Por esta razão, faz-se necessária a aplicação dos pré-tratamentos espectrais de modo a facilitar a interpretação das propriedades relacionadas aos sinais instrumentais. O alisamento ou suavização Savitzky-Golay foi utilizado para remoção dos espectros a presença de ruídos instrumentais aleatórios, aumentando assim, a razão sinal/ruído (Neves et al., 2012). A correção de espalhamento multiplicativo de luz, MSC, foi realizada devido às dispersões de luz em todas as direções (Javidnia et al., 2013). Finalmente, realizou-se também sobre os espectros o cálculo da primeira derivada, visando o ajuste da linha de base (Rantanen et al., 2000). A Figura 3 mostra os espectros NIR após a aplicação dos préprocessamentos.



Figura 2 - Espectro médio original das 24 amostras de jambo



Figura 3 - Espectros NIR após a etapa de préprocessamento (Suavização, MSC e $1^{\text {a }}$ derivada Savitzky-Golay).

Conforme pode ser observado também na Figura 2, os espectros NIR apresentam bandas tipicamente largas, resultado da ocorrência de bandas de combinação e por sobretons oriundos das ligações dos grupos $\mathrm{C}-\mathrm{H}, \mathrm{N}-\mathrm{H}, \mathrm{S}-\mathrm{H}$ e O-H. Uma característica marcante neste espectro NIR é a forte absorção referente a água presente nas ameixas, e que pode ser atribuída aos picos de $1450 \mathrm{~nm}$, primeiro sobretom, e $1940 \mathrm{~nm}$, combinação das vibrações O-H da água. Além da absorção da água é possível fazer atribuições da absorção referente aos açúcares contidos na fruta e que podem estar relacionados às bandas em $1160 \mathrm{~nm}$, influência do terceiro sobretom $\mathrm{C}-\mathrm{H}$, e a banda em $1780 \mathrm{~nm}$, devido ao primeiro sobretom (Inácio et al., 2013).

Após a etapa de pré-processamento espectral, a matriz de dados de jambo juntamente com os valores fornecidos pelos métodos de referências, foram desenvolvidos os modelos de calibração multivariada. O propósito da calibração multivariada é estabelecer uma relação matemática quantitativa entre os dados de saída de um instrumento e o parâmetro físico ou químico de interesse (Ferreira et al., 1999). Dentre os vários métodos matemáticos existentes para a realização da calibração, o PLS é o método mais usado. O PLS2 é uma versão do método PLS, no qual várias variáveis (ou parâmetros) dependentes são modeladas simultaneamente (Beebe et al., 1998). Em seguida, as amostras foram divididas nos conjuntos de calibração (2/3) e validação externa (1/3) 
utilizando-se o algoritmo SPXY (Galvão et al., 2005). O conjunto de calibração para ambos os parâmetros aplicando o PLS2 foi otimizado em função de três fatores: i) número de variáveis latentes; ii) coeficiente de calibração; iii) erro médio quadrático do conjunto de calibração (RMSEC). O melhor PLS2 obtido para o parâmetro AT foi utilizando 5 variáveis latentes, coeficiente de correlação de 0,90 e 20,71 de RMSEC. Para este modelo, o erro médio quadrático do conjunto de validação (RMSEP) foi de 22,34 e um coeficiente de correlação de 0,85 com 5 variáveis latentes. Este modelo não apresentou diferenças significativas entre os valores obtidos pelo método de referência segundo um teste t-pareado (95\% de confiança) (Derenne et al., 2011). A Figura 4-a mostra um bom ajuste entre os valores de AT experimentais versus os valores previstos pela técnica NIR, tanto para as amostras de calibração como previsão.



(a)

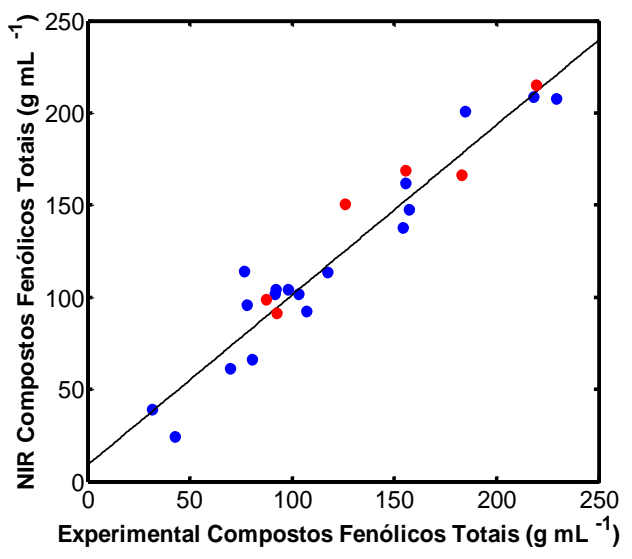

(b)

Figura 4 - Concentração prevista versus concentração medida experimentalmente pelo método de referência das amostras de calibração e validação (a) para antocianinas totais (AT) e (b) para compostos fenólicos totais (CFT) em jambos usando PLS2. (•) Calibration set; (•) Validation set.

Por outro lado, o melhor PLS2 obtido para o parâmetro CFT foi utilizando 8 variáveis latentes, coeficiente de correlação de 0,92 e 12,50 de RMSEC. Para este modelo, o erro médio quadrático do conjunto de validação (RMSEP) foi de 15,07 e um coeficiente de correlação de 0,92 com 8 variáveis latentes. Este modelo também não apresentou diferenças significativas entre os valores obtidos pelo método de referência segundo um teste t-pareado (95\% de confiança). A Figura 4-b mostra um bom ajuste entre os valores de CFT experimentais versus os valores previstos pela técnica NIR, tanto para as amostras de calibração como previsão.

\section{CONCLUSÃO}

Neste trabalho, um método rápido e não destrutivo para determinar simultaneamente o teor de 
antocianinas totais e os compostos fenólicos totais utilizando jambos intactos utilizando espectroscopia NIR e calibração multivariada foi apresentada. Como base nos resultados descritos para ambos os parâmetros, podemos concluir que a espectroscopia NIR juntamente com PLS2 pode ser utilizada na determinação destes parâmetros em jambos de forma intacta.

\section{REFERÊECIAS}

AZEVEDO, J. C. S. de. Estratégias de Obtenção do Corante do Jambo-Vermelho (Syzygium malaccense) e Avaliação de sua Funcionalidade. CT - Programa de Pós-graduação em Engenharia Química (Dissertações), 2010.

BEDNÁR, P.; PAPOUSKOVA, B.; MÜllER, L.; BARTAK, P.; STÁVEK, J.; PAVLOUSEK, P.; \& LEMR, K. Utilization of capillary electrophoresis/mass spectrometry (CE/MSn) for the study of anthocyanin dyes. Journal of Separation Science, 28, 1291-1299, 2005.

BEEBE, K.R.; PELL, R.J.; SEASHLTZ, M.B. Chemometrics: a practical guide. Wiley Interscience Publication, New York, 1998.

BRITO, P.K.S.; AZEVEDO, J.C.S.; DUARTE, M.M.L.; MATA, A.L.M.L. Estudo do efeito de parâmetros no processo de extração do corante de jambo. XX Congresso de Iniciação Científica Universidade Federal do Rio Grande do Norte - UFRN, 2009.

CHEPLICK, S.; KWON, Y; BHOWMIK, P.; SHETTY, K. Phenolic-linked variation in strawberry cultivars for potential dietary management of hyperglycemia and related complications of hypertension. Bior. Tech., v. 101, p. 404-413, 2010.

COSTA, R. C.; de LIMA, K. M. G. Prediction of parameters (soluble solid and pH) in intact plum using NIR spectroscopy and wavelength selection. J. Braz. Chem. Soc., vol.24, no.8, São Paulo, Aug. 2013.

DERENNE, A.; GASPER, R.; GOORMAGHTIGH, E. The FTIR spectrum of prostate cancer cells allows the classification of anticancer drugs according to their mode of action. Analyst 136, $1134-41,2011$.

FERREIRA, M. M. C.; ANTUNES, A. M.; MELGO, M. S.; VOLPE, P.L.O. Quimiometria I: calibração multivariada, um tutorial. Quim. Nova 22, 724-731, 1999.

GAlvão, R. K. H.; ARAujo, M. C. U.; JOsÉ, G. E., PONTES, M. J. C., Silva, E. C., SALDANHA, T.C.B. A method for calibration and validation subset partitioning. Talanta 67, 736-40, 2005.

GIUSTI, M.; WROLSTAD, R. E. Characterization and Measurement of Anthocyanins by UV-Visible Spectroscopy. New York: John Wiley \& Sons. (Current Protocols in Food Analytical Chemistry), 2001. 
INÁCIO, M. R. C., DE LIMA, K.M.G., LOPES, V. G., PESSOA, J. D. C., DE ALMEIDA TEIXEIRA, G.H. Total anthocyanin content determination in intact açaí (Euterpe oleracea Mart.) and palmitero-juçara (Euterpe edulis Mart.) fruit using near infrared spectroscopy (NIR) and multivariate calibration. Food Chem. 136, 1160-4, 2013.

JAVIDNIA, K.; PARISH, M.; KARIMI, S.; HEMMATEENEJAD, B. Discrimination of edible oils and fats by combination of multivariate pattern recognition and FT-IR spectroscopy: a comparative study between different modeling methods. Spectrochim. Acta. A. Mol. Biomol. Spectrosc. 104, 175-81, 2013.

LOPES, T. J.; XAVIER, M. S.; QUADRI, M. G.; QUADRI, M. B. Antocianinas: uma breve revisão das características estruturais e da estabilidade. R. Bras. Agrociência, Pelotas, v.13, n.3, p. 291-297, jul-set, 2007.

MISSANG, C. E.; GUYOT, S., \& RENARD, C. M. G. C. Flavonols and anthocyanins of bush butter, Dacryodes edulis (G. Don) H.J. Lam, fruit. Changes in their composition during ripening. Journal of Agricultural and Food Chemistry, 51, 7475-7480, 2003.

NEVES, A. C. O.; SOARES, G. M.; DE MORAIS, S. C.; DA COSTA, F. S. L., PORTO, D. L.; DE LIMA, K. M. G. Dissolution testing of isoniazid, rifampicin, pyrazinamide and ethambutol tablets using near-infrared spectroscopy (NIRS) and multivariate calibration. J. Pharm. Biomed. Anal. 57, 115-9, 2012.

RANTANEN, J.; ANTIKAINEN, O.; MANNERMAA, J.P.; YLIRUUSI, J. Use of the nearinfrared reflectance method for measurement of moisture content during granulation. Pharm. Dev. Technol. 5, 209-17, 2000.

RIBEIRO, E.P.; SERAVALLI, E.A.G. Química de alimentos, $2^{a}$ ed. São Paulo, Ed. Edgard Blucher, 184p., 2007.

SILVA, G. F. B.; DUART, M. M. L.; RIBEIRO, V. T.; MATA, A. L. M. L.; SOUZA, M. O. A. Análise da atividade antioxidante, compostos fenólicos totais e antocianinas monoméricas totais em extratos de jambo. XXIII CIC Congresso de Iniciação Científica, 2012.

WILLIAMS, C. A.; GREENHAM, J.; HARBORNE, J. B.; KONG, J. M.; CHIA, L. S.; GOH, N. K.; SAITO, N.; TOKI, K.; \&TATSUZAWA, F. Acylated anthocyanins and flavonols from purple flowers of Dendrobium c. 'Pompadour', Biochemical Systematics Ecology, 30, 667-675, 2002.

ZUDE, M.; PFLANZ, M.; DOSCHE, K.; SPINELLI, L.; \& TORRICELI, A. Non-destructive analysis of anthocyanins in cherries by means of Lambert-Beer and multivariate regression based on spectroscopy. Journal of Food Engineering, Engineering, 103, 68-75, 2011. 